\title{
Growth Hormone/Insulin Growth Factor-1 and Growth in IDDM Children and Adolescents
}

\author{
Laila M. Hanna, * Salwa M. El-Shebini, Salwa T. Tapozada, \\ Lobna A. GHATTAS, and Ashraf AMIN \\ National Research Center, Department of Nutrition, Cairo, Egypt
}

(Received June 14, 1999)

\begin{abstract}
Summary Diabetes mellitus is a syndrome of disturbed energy homeostasis caused by a deficiency of insulin or of its action and resulting in abnormal metabolism of carbohydrate, protein, and fat. The aim of this study was to study, by use of different anthropometrical parameters, the effect of diabetes mellitus either controlled or non-controlled on the growth pattern of the Egyptian children. At the same time, changes in the growth hormone/insulin-like growth factor-1 (GH/IGF-1) axis in the studied groups were investigated. The study was carried out on 50 healthy normal children and 127 diabetic children with insulin-dependent diabetes mellitus (IDDM) aged $2-18$ years. They were classified according to their fasting blood glucose level into controlled $<150 \mathrm{mg} / \mathrm{dl}$ and noncontrolled $150-300 \mathrm{mg} / \mathrm{dl}$. Then they were subdivided according to their age into three subgroups, $2-9,10-14,15-18$ years. At the start of the study the 2-9-year-old children had been diabetic since $1.8 \pm 0.32$ years and the 10-18-year-old children, since $3.96 \pm 0.12$ years. Clinical and some anthropometrical measurements were made including height and weight; and from them the body mass index (BMI) and Z-score for height and weight were calculated. In addition, mid-arm, wrist, head, and chest circumferences were measured. Radio-immunoassay (RIA) techniques for estimation of serum GH and IGF-1 were performed. The results obtained in this study indicated that the longitudinal growth was the parameter, which was more affected in the IDDM children, especially in those with noncontrolled disease. The mean \pm SEM of the Z-score for their height was $-1.16 \pm 0.27,-1.047 \pm 0.18$, and $-1.68 \pm 0.019$ for the age groups $2-9$, 10-14, and 15-18 years, respectively. The GH/IGF-1 axis was distributed, and $\mathrm{GH}$ concentrations were found to be numerically higher in the different age groups of the non-controlled diabetic children than in those of the normal children. Serum IGF-1 levels were significantly reduced
\end{abstract}

* To whom correspondence should be addressed. 
among the diabetic patients when compared with those recorded among their counterpart normal children. The reduction in the IGF-1 levels was pronounced in the non-controlled patients. A significant positive correlation was observed between all the anthropometric measurements and IGF-1 concentrations of the adolescents non-controlled diabetic children aged 10-14 years, defining its role in their growth. Thus the disturbed $\mathrm{GH} / \mathrm{IGF}-1$ axis in IDDM children and adolescents might contribute to their growth failure, mainly in the non-controlled patients.

Key Words: insulin-dependent diabetes mellitus, growth, growth hormone, insulin-like growth factor-1

Results based on bivariant analysis of height and weight in children with insulin-dependent diabetes mellitus (IDDM) clarified that reduced longitudinal growth was found despite modern therapeutic regimens [1]. Another study reported that, there is some interference in the growth of children with IDDM independent of the degree of metabolic control achieved; however, this interference does not seem to have a significant influence on final height [2]. On the other hand, it was concluded that diabetic children who maintained a higher degree of control grew and matured at the normal rate; in addition, all diabetic children having the lower degree of control showed accelerated growth after attaining a higher degree of control [3]. In children with diabetes, divergence exists between growth hormone $(\mathrm{GH})$ secretion and insulin-like growth factor-1 (IGF-1) production; and these abnormalities revert toward normal when metabolic control is improved [4]. Insulin is a regulator that improves the GH/IGF-1 axis, and endogenous insulin secretion allows sufficient IGF-1 production by the liver to maintain somatostatin hormone tone on somatotrophs [5]. The association between elevated circulating $\mathrm{GH}$ concentration and some of the long-term complications observed in patients with diabetes mellitus, e.g., poor growth in children and adolescents and microangiopathy in adults, has been reported $[6,7]$.

The aim of this work was to investigate the effect of diabetes mellitus on the growth pattern of Egyptian children aged 2-18 years. In addition, the effect of metabolic control on concentration of growth hormone and IGF-1 was investigated.

\section{SUBJECTS AND METHODS}

The study was carried on 127 IDDM children aged 2-18 years. They were chosen from the Diabetes Mellitus Institute, Cairo, Egypt. The samples were matched with 50 healthy normal children at the same age and the same socioeconomic status. Both groups were divided into subgroups according to their age, namely $2-9,10-14,15-18$ years. At the start of the study the 2-9-year-old children had been diabetic since $1.81 \pm 0.32$ years of duration; and the 10-18-year-old 
children since $3.96 \pm 0.12$ years of duration. The diabetic children were further classified according to their blood sugar level: a controlled group, whose blood sugar level was $<150 \mathrm{mg} / \mathrm{dl}$, and a non-controlled group, who had a blood sugar level of $150-300 \mathrm{mg} / \mathrm{dl}$.

The groups were subjected to detailed history and clinical examination to exclude any other organic or metabolic disorders that might affect the parameters to be investigated.

Some relevant anthropometrical measurements were recorded by use of standard methods [8]. The measurements included height and weight and head, chest, mid-arm, and wrist circumferences. Body mass index (BMI) (weight $\mathrm{kg} / \mathrm{height}^{2}$ ) and Z-score for height and weight were calculated [9].

Fasting blood samples were collected from each subject. Blood glucose was estimated by the Asatoor and King method [10], then the serum was separated and stored at $-30^{\circ} \mathrm{C}$ for estimation of $\mathrm{GH}$ and IGF-1 levels. $\mathrm{GH}$ determinations were carried out with a kit provided by Diagnosis Products Corporation (DPC, Los Angeles, U.S.A.), whereas IGF-1 determinations were made with a kit provided by Medgenic (code: 3015840, Zoning Industrial, B6220 Fleurus, Belgium).

Statistical analysis. The Z-score was calculated according to the methods of the World Health Organization (WHO) [9]. The analysis of variance (ANOVA) was calculated with EPi Info 6 system software, Center for Disease Control and Prevention (CDC), Atlanta, GA, U.S.A.

Linear regression analysis was used to define correlations of the hormone values and different anthropometrical and biochemical parameters.

\section{RESULTS}

Table 1 compares the means of some anthropometrical parameters by ANOVA. Of significance $p<0.02$ was the Z-score for weight in the non-controlled 2-9-year-olds as compared with the normals, whereas the score for the non-controlled 15-18-year-olds was lower $(p<0.05)$ than that of the counterpart controlled diabetics. Significant differences were also detected by the Z-score for height, which was lower $(p<0.05)$ for both age subgroups of $2-9$ and $15-18$ years as compared with the scores of their respective normals. Other significant differences were detected only in the 2-9-year-old subgroup where the BMI $(p<0.001)$, wrist $(p<0.01)$, head $(p<0.02)$, and chest $(p<0.01)$ circumferences were higher in the controlled than in the normal; while the BMI $(p<0.01)$, wrist $(p<0.05)$, and head $(p<0.05)$ circumferences were lower in the non-controlled than in the controlled diabetics.

Table 2 shows the comparison of the means of the biochemical parameters by ANOVA. The value of the blood glucose became greater as the age increased in all groups. Blood glucose was significantly higher ( $p<0.05$ for 2-9-year-olds, $p<$ 0.02 for $10-14$-year-olds and $p<0.01$ for 15 -18-year-olds) in the non-controlled than in either the normal or the controlled, the increase being more prominent in Vol. 28, No. 2, 2000 
L.M. HANNA et al.

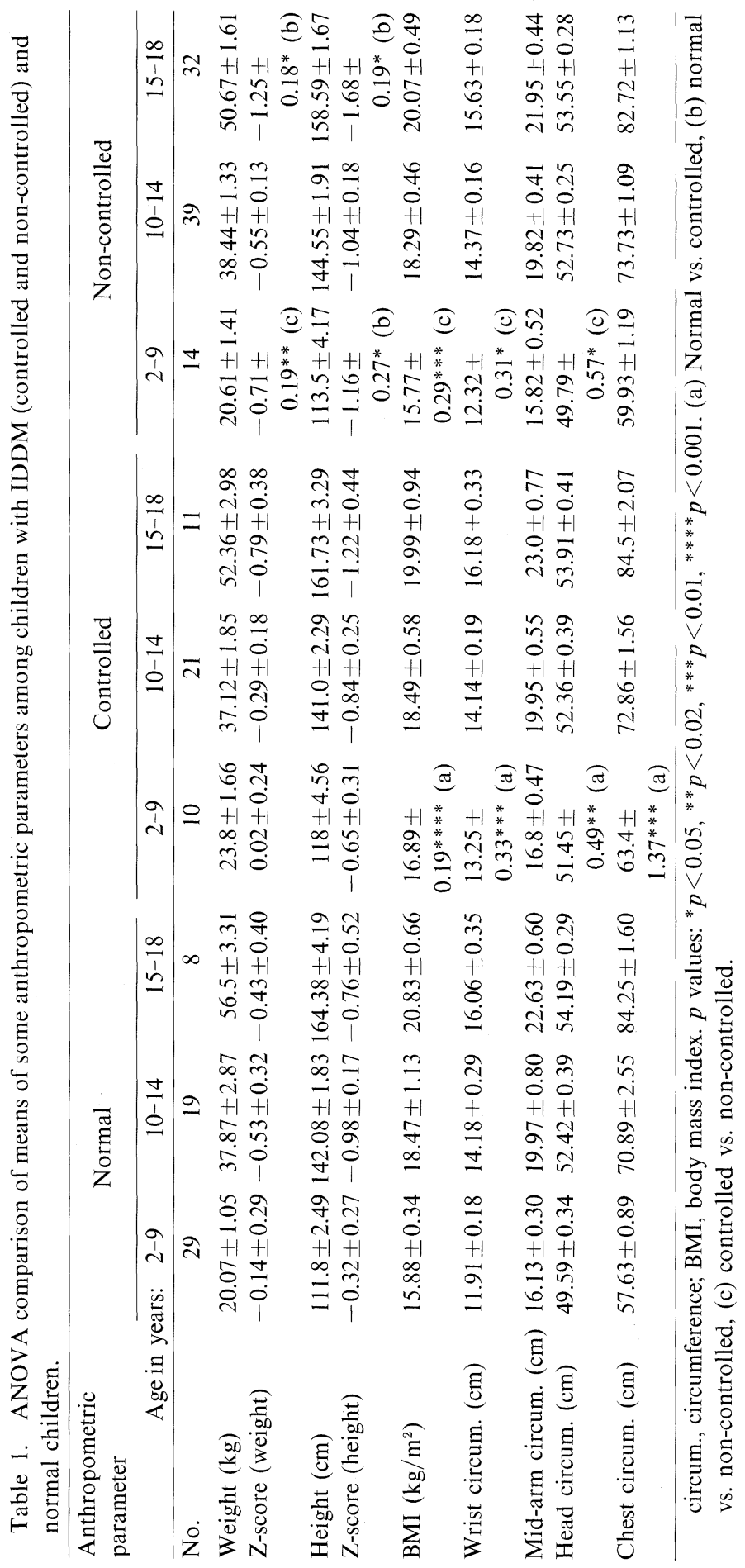

J. Clin. Biochem. Nutr. 

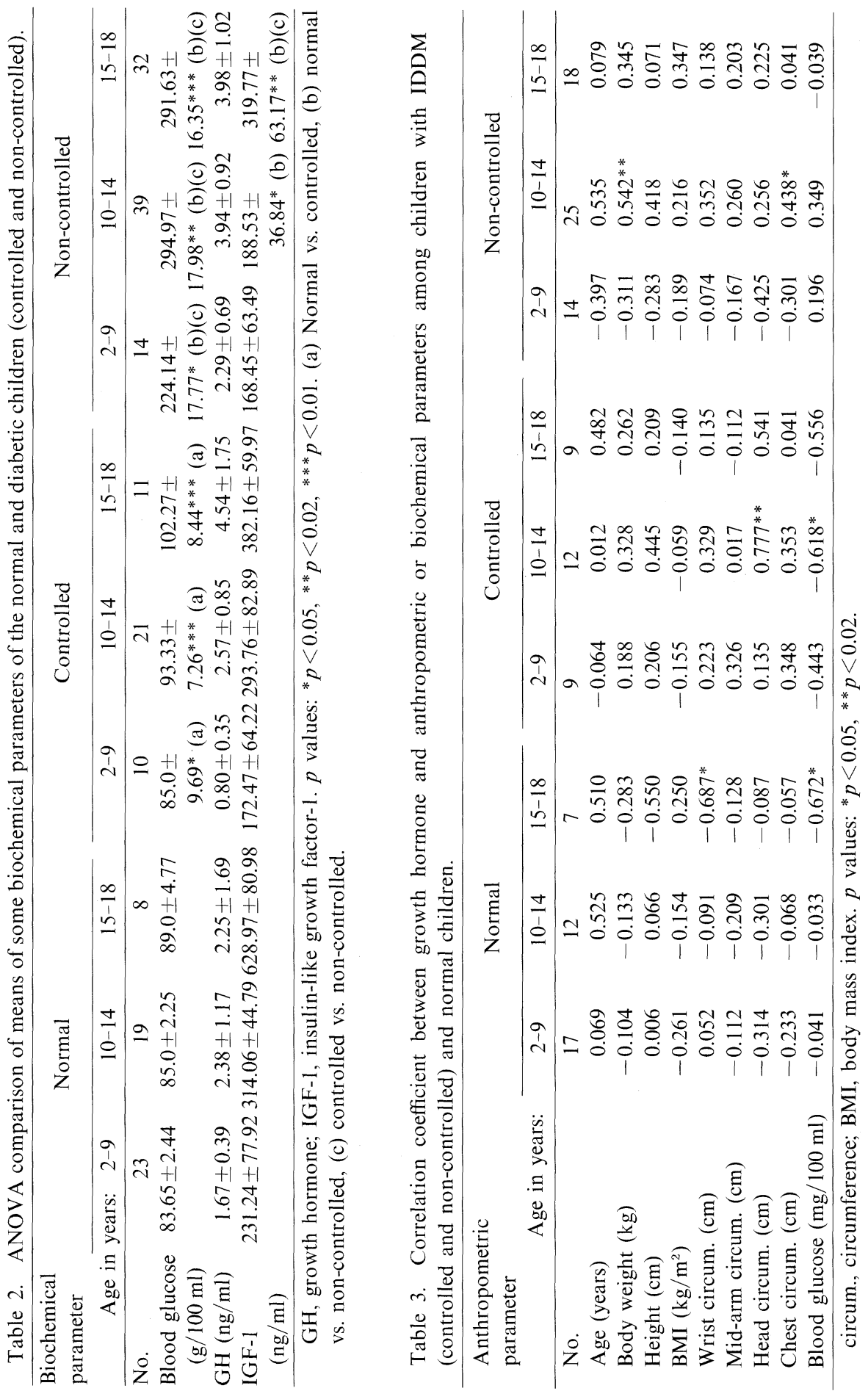

Vol. 28, No. 2, 2000 

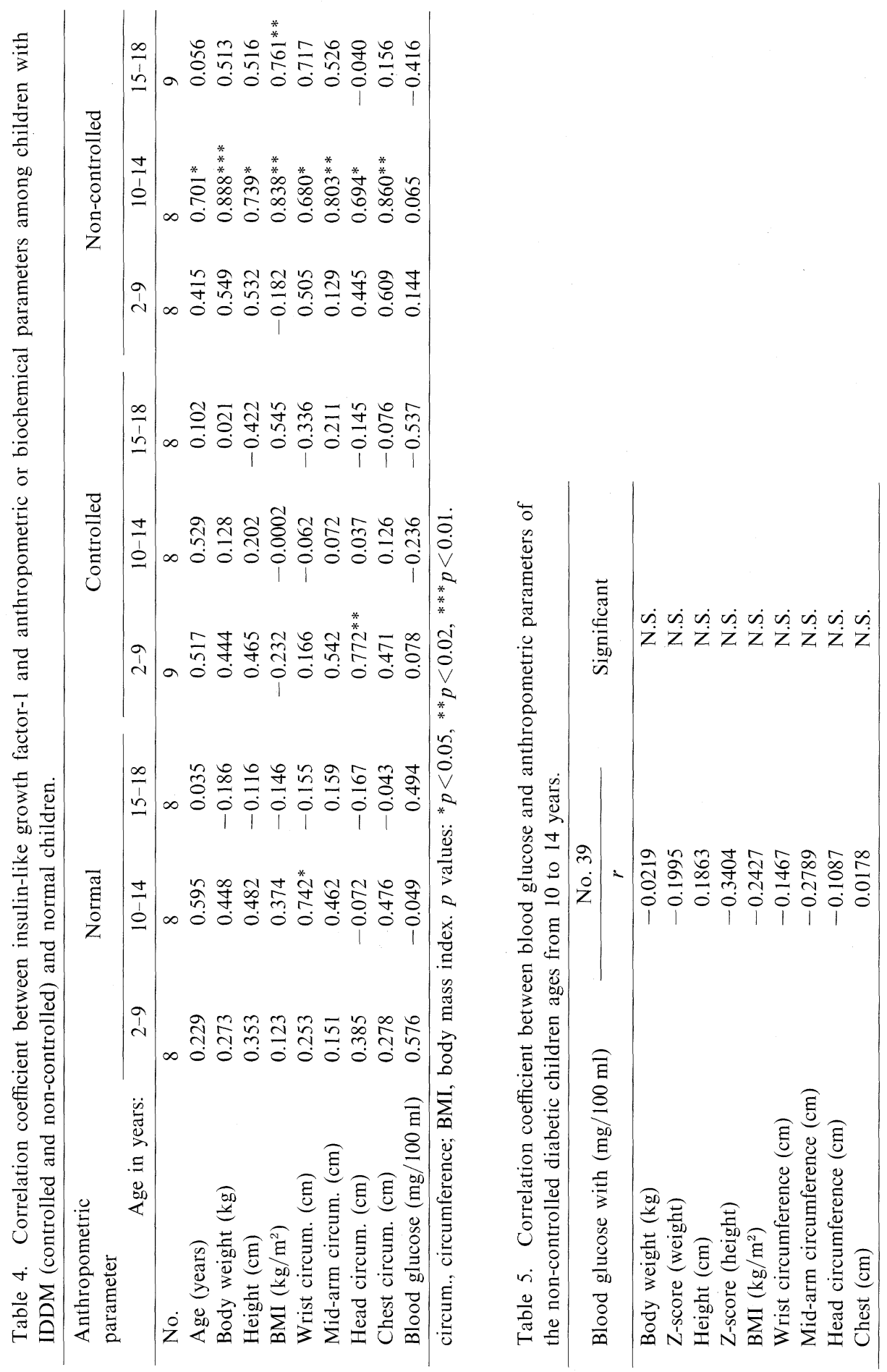

J. Clin. Biochem. Nutr. 
the two older age subgroups. Although there were no significant differences in the $\mathrm{GH}$ values between the groups, yet there was a numerical increase with age in the value for the controlled and non-controlled diabetics as compared with the normals. Lower values of IGF-1 were recorded for both diabetic groups, reaching a significant difference between the non-controlled diabetics and normal children at two ages $(p<0.05$ for $10-14$ years and $p<0.02$ for $15-18$ years).

Table 3 shows the correlation coefficients between GH and some anthropometrical and biochemical parameters. A positive correlation was recorded in the 10-14-year-old subgroup with the head circumference $(p<0.05)$ in the controlled group, and a negative one $(p<0.05)$ with blood glucose, in the controlled groups. Also, positive correlations were found with the body weight $(p<0.02)$ and chest circumference $(p<0.05)$ in the non-controlled group.

Table 4 shows the correlation coefficient between IGF-1 and the anthropometrical and biochemical parameters. A remarkable and consistent positive correlation was detected with all anthropometrical parameters and also with age, ranging from $p<0.05$ to $p<0.01$ in the 10 -14-year-old subgroup. This positive correlation was extended to the BMI $(p<0.02)$ of the $15-18$-year-olds of the non-controlled group. On the other hand, only a single positive correlation, with head circumference, was recorded for the controlled group.

Table 5 shows that there was no correlation between the blood glucose level and any of the anthropometrical parameters of the non-controlled diabetic children (10-14-year-olds).

\section{DISCUSSION}

Many studies demonstrated growth impairment in diabetic children regardless of the degree of metabolic control $[1,11]$, whereas other studies found no growth retardation $[12,13]$. In the present study the results showed that despite the fact that some of the calculated values for height in some age groups of diabetic children were high compared with the normal values, yet by using the corrected Z-score parameter for height, which bypasses the intragroup age variation, we found different degrees of retardation in height. This retardation was pronounced in the non-controlled diabetic children as well as in the adolescents 15-18 years of age in the controlled group. The $\mathrm{Z}$-score for body weight also revealed some degree of retardation, especially in the non-controlled adolescent patients 15-18 years of age. This decrease in body weight was proved by other means, i.e., by BMI, which reflects the adequacy of weight in relation to height. Values obtained in this study concerning the diabetic patients were comparable with the normal ones in spite of the shorter stature of the diabetic patients. However, two exceptions were observed: the first was a significantly high value of BMI obtained among the young controlled group $(p<0.01)$ which may denote that there was more weight gain among them, a phenomenon often accompanying intensive treatment regimens designed for IDDM patients [14]. In contrast a significantly low BMI value was found for Vol. 28, No. 2, 2000 
the similar age category of the non-controlled groups. It has been reported that untreated IDDM and nutritional deprivation share many metabolic similarities $[15]$.

Wrist and head circumferences, which indicate bone maturity, were significantly decreased in younger age groups of both controlled and non-controlled patients and not comparable to height growth. It was reported that in diabetic boys and girls skeletal maturity is disproportionately decreased in onset in relation to height onset [16].

Chest circumferences representing muscle development, and mid-arm circumferences, representing peripheral muscle and fat depots, showed slight numerical values slightly lower than normal in the 15-18-year-olds of the non-controlled group. In this respect, we suggest that somatic development as well as skeletal growth of the diabetic children, especially around puberty, was impaired and at the same time both processes were dependent on the metabolic control.

The results obtained in this study demonstrated that the serum GH concentration rose with age. This is in line with previous data showing that in normal individuals GH concentrations are higher in adolescence than in either childhood or adulthood [17]. The same trend was observed in both the controlled and non-controlled diabetic children but was more pronounced in the non-controlled group. Controlled and non-controlled 15-18-year-olds also had numerically higher levels of $\mathrm{GH}$ than their counterpart normals. The data reported here are consistent with those of other researchers, who reported that there was a maximal increase in serum GH concentration pulses during the late, but not early, pubertal stage, accompanied by an increase in the serum GH concentration interpulse baseline during the pubertal stage, in patients with IDDM under moderate glycemic control [18]. In patients with IDDM it is unknown whether GH resistance is a result of a receptor deficiency, an abnormal postreceptor pathway or both [19]. Some in vivo confirmation of these observations in humans assumed that the growth hormone binding protein (GHBP) was identical to the extracellular domain of the GH receptor [20]. Insulin therapy leads to an increase in GHBP, but these increased levels are less than those found in subjects without diabetes [21].

Alterations in IGF-1 were observed in this study in the diabetics of all different age groups. Their IGF-1 serum levels were reduced when compared with those recorded for their counterpart normal children, and significant differences were found for the age groups of 10-14 and 15-18 years. The reduction in the IGF-1 levels was more pronounced in the non-controlled patients than in the controlled ones. Previous studies reported different results regarding serum IGF-1 levels in children with IDDM and the effect of glycemic control on IGF-1. Serum IGF-1 levels correlated with serum glycosylated hemoglobin in some studies [22, $23]$, but not in others $[24,25]$. In this study no significant correlation was found between the IGF-1, or any of the anthropological parameters and the glucose levels in either diabetic group. Implication of hypoinsulinemia rather than hyper- 
glycemia as a responsible factor for the derangement of $\mathrm{GH} / \mathrm{IGF}-1$ axis has been reported [19]. Alterations in the IGF-1 observed in the present study are consistent with the hypothesis that these changes are an adaptation by which intracellular substrates may be used for the maintenance of basic cellular functions instead of growth under a catabolic state [15]. In this respect, a significant positive correlation was observed between the IGF-1 concentration and each of the anthropometrical measurements in the adolescent non-controlled diabetic children 10-14 years of age. Puberty is a period of development during which rapid growth occurs. Regulation of the IGF-1 concentration is disturbed in children with IDDM, particularly during adolescence [26].

In conclusion a better treatment regimen to reach optimal glycemia in IDDM patients is recommended. Besides glucose level is a biomarker for better general health of these patient, and monitoring glucose could be a helpful effective way to overcome the disturbance recorded in the GH/IGF-1 axis, especially in the non-controlled subjects, which may have contributed to their growth failure.

\section{REFERENCES}

1. Thon, A., Heinze, E., Feilen, K.D., Holl, RW., Schmidt, H., Koletzko, S., Wendel, U., and Nothjunge, J. (1992): Development of height and weight in children with diabetes mellitus. Report on two prospective multicenter studies, one cross-sectional and one longitudinal. Eur. J. Pediatr., 151, 258-262.

2. Garcia, R.V.G., Moreiras, M., Batista, J., Penas-Cortes, J., and Morales, M.J. (1990): Effect of childhood-onset insulin dependent diabetes mellitus on final height. Acta Pediatr. Scand. (Suppl.), 370, 207.

3. Jackson, R.L. (1984): Growth and maturation of children with insulin dependent diabetes mellitus. Pediatr. Clin. North Am., 31, 545-567.

4. Asplin, C.M., Faria, A.C.S., Carlsen, E.C., Vaccaro, V.A., Barr, R.E., Iranmanesh, A.L.T., Lee, M.M., Veldhuis, J.D., and Evans, W.S. (1989): Alterations in the pulsatile mode of growth hormone release in men and women with insulin-dependent diabetes mellitus. $J$. Clin. Endocrinol. Metab., 69, 239-245.

5. Counts, D.R., Gwirtsmen, H., Carlsson, M.S., Lesem, M., and Cutler, G.B. (1992): The effect of anorexia nervosa and refeeding on growth hormone binding protein, the insulin like factor (IGFs) and the IGF-binding proteins. J. Clin. Endocrinol. Metab., 75, 762-767.

6. Mauras, N., Merimee, T.J., and Rogol, A.D. (1991): Function of the growth hormone IGF-1 axis in the profoundly growth retarded diabetic child. Evidence for defective target organ responsiveness in the Mauriac syndrome. Metabolism, 40, 1106-1111.

7. Selby, J.V., Fitzsimmons, S.C., Newman, J.M., Katz, P.P., Sepe, S., and Showstack, J. (1990): The natural history and epidemiology of diabetic nephropathy. Implications for prevention and control. JAMA, 263, 1954-1960.

8. Jelliffe, D.B. (1966): The Assessment of the Nutritional Status of the Community, Vol. 63, World Health Organization, Geneva.

9. WHO (1963): World Health Organization Measuring Change in Nutritional Status.

10. Asatoor, A.M., and King, E.J. (1954): Determination of blood glucose. Biochem. J., 56, Xliv.

11. Brown, M., Ahmed, M.L., Clayton, K.L., and Dunger, D.B. (1994): Growth during childhood and final height in type 1 diabetes. Diabetic Med., 11, 182-187.

12. Clarson, C., Daneman, D., and Ehrlich, R.M. (1985): The relationship of metabolic control to growth and pubertal development in children with insulin-dependent diabetes. Diabetes Res. Clin. Exp., 2, 237-241.

Vol. 28, No. 2, 2000 
13. Batch, J.A., and Werther, G.A. (1992): Changes in growth hormone concentrations during puberty in adolescents with insulin dependent diabetes. Clin. Endocrinol., 36, 411-416.

14. Leiter, L.A., Lukaski, H.C., Kenny, D.J., Barnie, A., Camelon, K., Ferguson, R.S., Maclean, S., Simkins, S., Zinman, B., and Ceary, P.A. (1994): The use of bioelectrical impedance analysis (BIA) to estimate body composition in the diabetes control and complications trial (DCCT). Int. J. Obes. Relat. Metab. Disord., 18, 829-835.

15. Bereket, A., Lang, C.H., Blethen, S.L., Marie, C.G., Fan, J., Frost, R.A., and Wilson, T.A. (1995): Effect of insulin on the insulin like growth factor system in children with new onset insulin dependent diabetes mellitus. J. Clin. Endocrinol. Metab., 80, 1312-1317.

16. Edelsten, A.D., Hughes, I.A., Oakes, S., Gordon, I.R.S., and Savage, D.C.L. (1981): Height and skeletal maturity in children with newly-diagnosed juvenile onset diabetes. Arch. Dis. Child., 56, 40-44.

17. Zadik, Z., Chalew, S.A., McCarter, J., Meistas, M., and Kowarski, A.A. (1985): The influence of age on the 24 hour integrated concentration of growth hormone in normal individuals. J. Clin. Endocrinol. Metab., 60, 513-516.

18. Edge, J.A., Matthews, D.R., and Dunger, D.B. (1990): The dawn phenomenon is related to overnight growth hormone release in adolescent diabetics. Clin. Endocrinol., 33, 729-738.

19. Mercado, M., Molitch, M.E., and Bumann, G. (1992): Low plasma growth hormone binding protein in IDDM. Diabetes, 41, 605-609.

20. Hochberg, Z., Hertz, P., and Colin, V. (1992): The distal axis of growth hormone in nutritional disorders: GH binding protein, insulin-like growth factor-1 (IGF-1) and (IGF-1) receptor in obesity and anorexia nervosa. Metabolism, 41, 106-112.

21. Arslanian, S.A., Menon, R.K., Gierl, A.P., Heil, B.V., and Foley, T.P., Jr. (1993): Insulin therapy increase low plasma growth hormone binding protein in children with new onset type 1 diabetes compared with puberty matched controls. Diabetes Med., 10, 833-838.

22. Winter, R.J., Phillips, L.S., Klein, M.N., Traisman, H.S., and Green, O.C. (1979): Somatomedin activity and diabetic control in children with insulin-dependent diabetes. Diabetes, 28, 952-954.

23. Blethen, S.L., Sargeant, D.T., Whitlow, M.G., and Santiago, J.V. (1981): Effect of pubertal stage and recent blood glucose control on plasma somatomedin in children with insulindependent diabetes mellitus. Diabetes, 30, 868-872.

24. Salardi, S., Cacciari, E., Ballardini, D., Reighetti, F., Capelli, M., Cicognani, A., Zucchini, S., Natali, G., and Tassinari, D. (1986): Relationships between growth factors (somatomedin-c and growth hormone) and body development, metabolic control, and retinal changes in children and adolescents with IDDM. Diabetes, 35, 832-836.

25. Rogers, D.G., Sherman, L.D., and Gabbay, K.H. (1991): Effect of puberty on insulin-like growth factor-1 and $\mathrm{Hb}-\mathrm{A}_{1}$ in type 1 diabetes. Diabetes Care, 14, 1031-1035.

26. Cinaz, P., Kendirci, M., Kurtoglu, S., Gokccora, N., Buyan, N., Yavuz, I., and Demir, A. (1996): Serum levels of insulin-like growth factor-1 and insulin-like growth factor binding protein-3 in children with insulin dependent-diabetes mellitus. J. Pediatr. Endocrinol. Metab., 9, 475-482. 